\title{
Application of fuzzy finite element method in addressing the presence of uncertainties
}

\author{
A.Y.N. Yusmye \\ Institute of Engineering Mathematics, Universiti Malaysia Perlis, Kampus Pauh Putra, Arau, Perlis, Malaysia
}

\author{
A.K. Ariffin, S. Abdullah \& S.S.K. Singh \\ Department of Mechanical and Materials Engineering, Universiti Kebangsaan Malaysia, UKM Bangi, Selangor, \\ Malaysia
}

M. Beer

Institute for Computer Science in Civil Engineering, Leibniz University, Hannover, Germany

\begin{abstract}
This research works is focused on the analysis of Fuzzy Finite-Element Method (FFEM) with the present of uncertainties. In considering a major engineering science problems, like damage processes or loading in consequence of real incident, uncertainty are present. Uncertainty is due to lack of data, an abundance of information, conflicting information and subjective beliefs. With that reason, the present of uncertainties is needed to avoid for prevent the failure of the material in engineering. The goals of this study are to analyzed and determine the application of FFEM by taking into consideration of the epistemic uncertainties involved toward the single edge crack plate and beam. Since it is crucial to develop an effective approach to model the epistemic uncertainties, the fuzzy system is proposed to deal with the selected problem. Fuzzy system theory is a non-probabilistic method, and this method is most appropriate to interpret the uncertainty compared to statistical approach when the deal with the lack of data. Fuzzy system theory contains a number of processes started from converting the crisp input to fuzzy input through fuzzification process and followed by the main process known as mapping process. In mapping process stage, the combination of fuzzy system and finite element method are proposed. In this study, the fuzzy inputs are numerically integrated based on extension principle method. Obtained solutions are depicted in terms of figures and tables to show the efficiency and reliability of the present analysis.
\end{abstract}

\section{INTRODUCTION}

An uncertainty is define as a gradual assessment of the truth content of a proposition, doubt arises as to whether the truth content may be stated with sufficient accuracy using each of the data models in all cases. Also, uncertainties are defined as the vagueness and lack of the information or data, Farkas (2010). The element of uncertainty is one of the biggest challenges in the field of engineering. $\mathrm{He}$ (2007) mentioned that, in general, uncertainty can divided into three types, which are stochastic uncertainty, epistemic uncertainty and error. Stochastic uncertainty is due to variations in the system. For the epistemic uncertainty, it exists as a result of incomplete information, ignorance and lack of knowledge caused by the lack of experimental data. When compare to the error, this uncertainty is the uncertainty that can be identified due to the imperfections in the modelling and simulation.

For a several decades ago, uncertainty is modelled according to the theory of probability. Probability method is very effective in solving the problem of stochastic uncertainty, but this method is not suitable to be used to solve problem involving the lack of data. Some scholars hold that the use of non-probability methods are most appropriate to interpret the uncertainty compared to statistical approach when deal with the lack of data. The interval analysis (Zimmermann 2012), convex modelling (Tapaswini et al. 2012) and fuzzy set theory (Ozkoka \& Cebi 2014) are the main categories of non-probabilistic methods. Specifically, fuzzy system is a system to be precisely defined and it applied all the theories that use the basic concept of fuzzy set theory. According to Savoia (2002), the main advantages of using the fuzzy set theory than the fuzzy probability theory is able to maintain the intrinsic random nature of the physical variables without the need for modeling the probability density function. The simple justification for fuzzy system theory is the real world is too complicated for precise explanation and description to be obtained. Fuzzy system theories are knowledge based or rule based systems. Fuzzy systems have been applied to a wide field. 
Finite Element Method (FEM) has become a powerful tool in solving numerous complex scientific and engineering problems. By using FEM, the complicated structured of any materials can be discretized into a small finite element. All the elements are assembling then applying the respective requirement to obtain the output. System parameters such as geometry, material properties, external load or boundary conditions are considered as crisp value or can be defined exactly in the convectional FEM. However, rather than the particular value, it may have only the vague, imprecise and incomplete information about the variables being a result. The present of uncertainty is the biggest challenging in engineering in order to deal with uncertainty in designing the material and cannot be avoided.

Fuzzy Finite Element Method approach (FFEM) is present to deal with the uncertainty and it is the merger method of fuzzy approach with the conventional Finite Element Method (FEM). In FFEM approach, conventional FEM is used as a parent in order to obscure the data input mapping to the output data (Behera, 2012). In this paper, there are two illustrative examples which applied the fuzzy finite element method with considering the presence of uncertainties. The first illustrative example is the application of FFEM in single edge crack plate by considering a fuzzy variable of crack length. And the second one is to analyze the structural reliability on beam.

\section{PRELIMINARIES}

In the following paragraphs, some of the notation, definition and preliminaries which are used further in this paper discuss deeply.

Definition 1:

A fuzzy number is convex normalized fuzzy set of the real line such that

$$
\mu_{A}=X \rightarrow[0,1]
$$

\section{Definition 2:}

We can defined an arbitrary triangular fuzzy number as $\tilde{A}=\left[a_{L}, a_{N}, a_{R}\right]$ and trapezoidal fuzzy number as $\tilde{B}=\left[b_{L}, b_{N L}, b_{N R}, \mathrm{~b}_{R}\right]$. The fuzzy number $\tilde{A}$ is said to be triangular fuzzy number when the membership function is given by

$$
\mu_{\tilde{A}}(x)= \begin{cases}0 & x \leq a_{L} \\ \frac{x-a_{L}}{a_{N}-a_{L}} & a_{L} \leq x \leq a_{N} \\ \frac{a_{R}-x}{a_{R}-a_{N}} & a_{N} \leq x \leq a_{R} \\ 0 & x \geq a_{R}\end{cases}
$$

The triangular fuzzy number $\tilde{A}=\left[a_{L}, a_{N}, a_{R}\right]$ can be transformed into interval form by using $\alpha$-cut as Equation (3).

$$
\begin{aligned}
\tilde{A} & =\left[a_{L}, a_{N}, a_{R}\right] \\
& =\left[a_{L}+\left(a_{N}-a_{L}\right) \alpha, a_{R}-\left(a_{R}-a_{N}\right) \alpha\right]
\end{aligned}
$$

The fuzzy number $\tilde{B}$ is said to be trapezoidal fuzzy number when the membership function is given by

$\mu_{\tilde{B}}(x)= \begin{cases}0 & x \leq b_{L} \\ \frac{x-b_{L}}{b_{N L}-b_{L}} & \mathrm{~b}_{L} \leq x \leq b_{N L} \\ \frac{b_{R}-x}{b_{R}-b_{N R}} & \mathrm{~b}_{N R} \leq x \leq b_{R} \\ 0 & x \geq b_{R}\end{cases}$

The trapezoidal fuzzy number $\tilde{B}=\left[a_{L}, a_{N}, a_{R}\right]$ can be transformed into interval form by using $\alpha$-cut as Equation (5).

$$
\begin{aligned}
\tilde{B} & =\left[b_{L}, b_{N L}, b_{N R}, b_{R}\right] \\
& =\left[b_{L}+\left(b_{N L}-b_{L}\right) \alpha, b_{R}-\left(b_{R}-b_{N R}\right) \alpha\right]
\end{aligned}
$$

\section{FUZZY FINITE ELEMENT METHOD}

The fuzzy finite element method is a combination of fuzzy approach and conventional finite element method. Figure 1 shows a flow chart for a fuzzy finite element process which included the fuzzification process, mapping process, $\alpha$-cut level process and ending with defuzzification process.

The procedure for the analysis by using the fuzzy finite element methods is started by transform the crisp input or real value for input into the fuzzy input uncertainty through the fuzzification process. The variable that have fuzzy uncertainty is probably the material properties, geometry of the materials, boundary condition or loading (Akapan 2001). Triangular fuzzy numbers are used for understanding the function of all parameters of fuzzy membership. This FFEM approach continues with the mapping process, where the fuzzy input will be used in the FEM model by using $\alpha$ -cut level. Vertex method is common numerical methods used in the implementation of the extension principle. Vertex method is a combination of interval arithmetic methods with $\alpha$-cut method. The purpose of this mapping is to map two or more fuzzy input to the fuzzy output with binary combinations of as many as where is the number of fuzzy input parameters. Figure 2 showed the example of $\alpha$-cut method for fourth level of alpha for each variable available. 


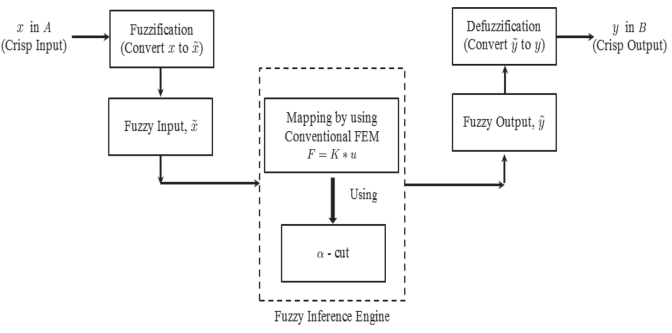

Figure 1. Flow chart for FFEM.

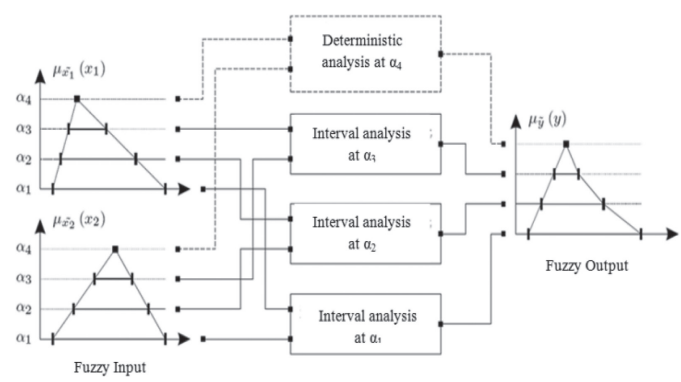

Figure 2. $\alpha$ - cut method for each alpha level Source: Farkas et al. (2010).

After the mapping process, the new probability distributions of the fuzzy output are created. The final stage in FFEM is defuzzification process in which stress intensity factor is the final output for this study. In defuzzification process, there are many different methods available which is center of gravity, center of area, mean of maximum, middle of maximum, fuzzy mean, fuzzy clustering defuzzification and so many. The center of gravity or centroid (COG) is the familiar defuzzification method used by many researchers and in this study. Defuzzification process is the process that transforms the fuzzy output into crisp output. This technique determines the point at which it will distribute one area into two parts which have the same value.

\section{RESULT AND DISCUSSION ON ILLUSTRATIVE EXAMPLE}

\subsection{A single edge crack plate by considering the fuzzy variable of crack length}

The model used in this study is Aluminium Alloy 2024-T351, since this material widely used in structural engineering such as parts of the hydraulic valve and piston, gear and shafts. In this study, three parameters that are Young's modulus, $E$,
Poisson ratio, $v$ and Density, $\rho$ of Aluminium 2014T3, are used as non-fuzzy parameters. Compare to the crack length, $a$ is treated as a fuzzy parameter. The geometry used in this study is based on the stress analysis of crack handbook by Tada et. al (2000) as shown in Figure 3.

From analytical aspect, the stress intensity factor under mode I can be obtained by using the Equation (6) as below:

$$
K_{I}=\sigma \sqrt{\pi} a F(a / b)
$$

The geometry function $F(a / b)$ in equation (6) can be calculated by using the equation (7).

$$
\begin{aligned}
F(a / b)= & 1.122-0.231(a / b)+10.55(a / b)^{2} \\
& -21.7(a / b)^{3}+30.382(a / b)
\end{aligned}
$$

where $a$ is the crack length, $b$ is width of the geometry, $\pi$ is the constant with the value 3.1415927 and $\sigma$ is the applied stress. The ratio of $a / b$ for the geometry in Figure 3 is 0.1 . The fuzzy value of crack length, $a$ is represent in a trapezoidal fuzzy

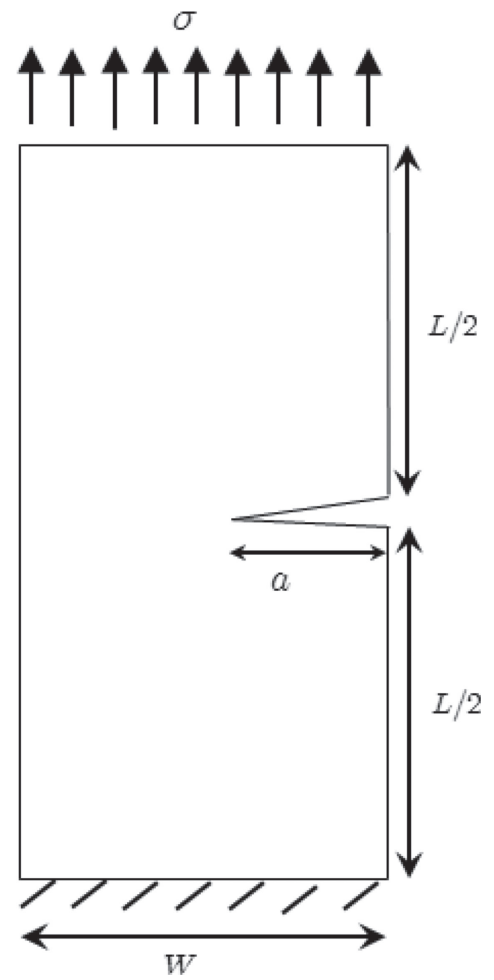

Figure 3. Two-dimensional loaded plate. 
number in form as $a=\left(a_{1}, a_{2}, a_{3}, a_{4}\right)$ and written in $\alpha$-cut form and finally the interval fuzzy number can written as Equation 8.

$$
\left[a_{L}, a_{R}\right]=\left[a_{1}+\left(a_{2}-a_{1}\right) \alpha, a_{4}-\left(a_{4}-a_{3}\right) \alpha\right]
$$

As mentioned early, this study only considered the fuzzy variable of crack length whereas the Young's Modulus and Poisson Ratio are considered as crisp variables.

Using fuzzy values are present the result in interval form and sketch it in trapezoidal membership function graph. The fuzzy number for crack length are $a=(0.1,0.32,0.41,0.49)$. By using Equation 6 and 7, the fuzzy intervals in term of $\alpha$-cut are obtained as shown in Table 1. These fuzzy intervals are substitute into SIF formula in term of interval as

$$
\begin{aligned}
& K_{I(R)}=\sigma \sqrt{\pi} a_{(R)} F(a / b) \\
& K_{I(L)}=\sigma \sqrt{\pi} a_{(L)} F(a / b)
\end{aligned}
$$

and continue till all SIF are obtained. Where $\left.K_{I(L)}\right)$ and $\left.K_{I(R)}\right)$ is a left and right value of interval respectively for each $\alpha$-value. The output for SIF with the fuzzy crack length is shown in Table 2. Beside the result is shown in Figure 4. The cases of crack length are treating as interval at a width again increases as we increase the number of elements. In fuzzy, the large width of interval for membership functions is giving more accurate result.

The result is shown in Figure 4 depicts the SIF plot the single edge crack plate. It may be seen from the above numerical result that the natural stress (crisp values) are constant with increase in number of elements as it should be even though only two elements consider here. However it

Table 1. Fuzzy value of crack length for each $\alpha$-level.

\begin{tabular}{lll}
\hline Beta $(\alpha$-Level $)$ & $a_{L}$ & $a_{R}$ \\
\hline 0 & 0.1 & 0.49 \\
0.1 & 0.122 & 0.482 \\
0.2 & 0.144 & 0.474 \\
0.3 & 0.166 & 0.466 \\
0.4 & 0.188 & 0.458 \\
0.5 & 0.21 & 0.45 \\
0.6 & 0.232 & 0.442 \\
0.7 & 0.254 & 0.434 \\
0.8 & 0.276 & 0.426 \\
0.9 & 0.298 & 0.418 \\
1 & 0.32 & 0.41 \\
\hline
\end{tabular}

Table 2. Interval value of SIF for each $\alpha$-level.

\begin{tabular}{lll}
\hline Beta $(\alpha$-Level $)$ & $a_{L}$ & $a_{R}$ \\
\hline 0 & 6.40 & 14.16 \\
0.1 & 7.06 & 14.04 \\
0.2 & 7.67 & 13.92 \\
0.3 & 8.24 & 13.81 \\
0.4 & 8.77 & 13.69 \\
0.5 & 9.27 & 13.57 \\
0.6 & 9.74 & 13.45 \\
0.7 & 10.19 & 13.32 \\
0.8 & 10.63 & 13.20 \\
0.9 & 11.04 & 13.08 \\
1 & 11.44 & 12.95 \\
\hline
\end{tabular}

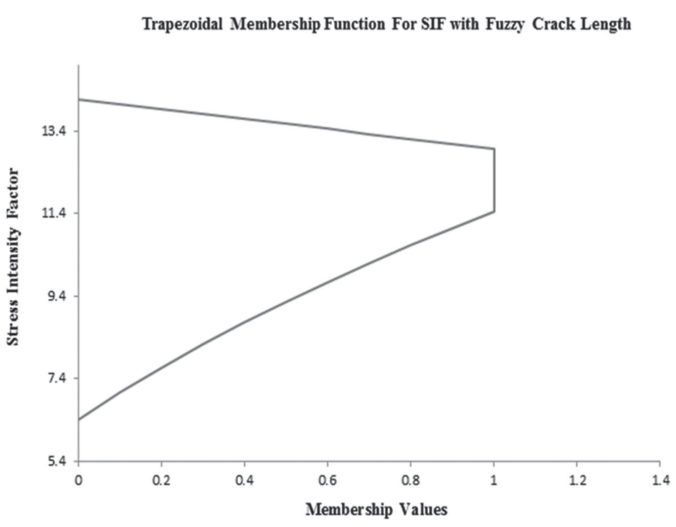

Figure 4. Trapezoidal membership function for SIF.

differs for values got in each element by using fuzzy method. The cases of crack length are treating as interval at a width again increases as we increase the number of elements. In fuzzy, the large width of interval for membership functions is giving more accurate result.

\subsection{Analysis on beam structure}

The analysis on structural reliability in the presence of uncertainties is performed in beam structure. In the analysis of beam structure in Figure 5, the moment of inertia and modulus of elasticity of beam are considered as fuzzy input parameters with incomplete data.

The existing data shows the moment of inertia is a normal distribution with mean value $6.58 \times 10^{-5} \mathrm{~m}^{4}$ and constant variance, COV of 0.1 , while the elastic modulus has a normal distribution with mean and COV value of 0.1 and $73.1 \mathrm{GPa}$ respectively. The uniformly distributed load applied to the beam is considered as an input that has no data. Thus, the opinion of an experienced 


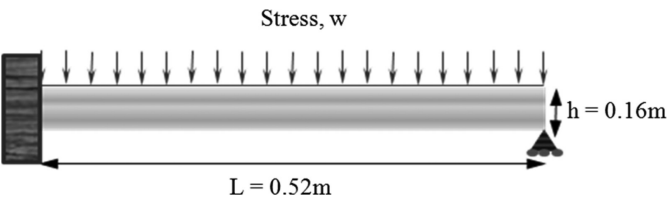

Figure 5. The geometry of the beam structure.

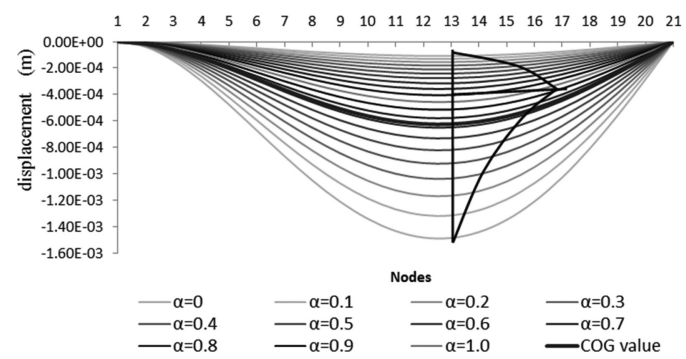

Figure 6. Fuzzy outputs of displacement using COG method.

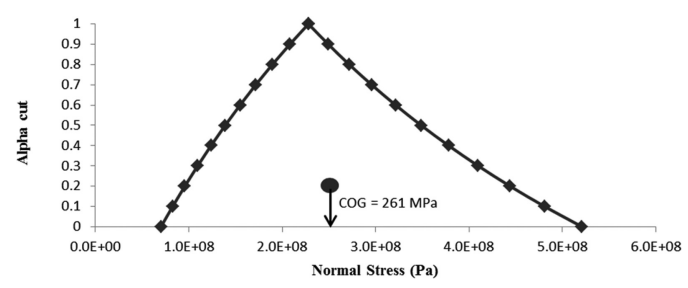

Figure 7. Fuzzy outputs of normal stress using COG method.

specialist should be considered to determine the likely distribution of that load. In this study, the distributed load is considered as a normal distribution with mean value $5.55 \mathrm{MN} / \mathrm{m}$ and $\mathrm{COV}$ value of 0.2 . The base area of membership function for the three fuzzy input parameters considered to have the interval of 6 standard deviation and $\sigma$ normal distribution, where $99 \%$ of the distribution included in the analysis.

In general, the deformation and stress in the beam plays an important role in determining the reliability of the structure. The output at each node in the FFEM is in membership function form. A COG value at each node is calculated with the centroid techniques used in the defuzzification technique. The maximum displacement is $0.00062 \mathrm{~m}$ at node 13. COG values in Figure 6 and Figure 7 represent the FFEM results considering uncertainties in the input parameters. In the reliability analysis of beam structures, the critical parameter is the stress. The COG value for the maximum normal stress is at node 1 with the value $261 \mathrm{MPa}$ and this value is less than the yield strength of a material. The results showed that the reliability of the structure under this illustrative example is 0.9733 , which mean it is close to 1. Therefore, the beam structure is still in a safe region of the elastic deformation range. Besides that, the maximum normal stress value for conventional FEM is $228 \mathrm{MPa}$. From this, it shown that the FFEM method was produced more conservative value of reliability compare to conventional FEM method.

\section{CONCLUSION}

Obtained solutions are depicted in terms of figures and tables to show the efficiency and reliability of the present analysis. This study shows that, the FFEM approach is conservative method to solve the uncertainties problems. One way to reduce the uncertainty in the data is by experiment. FFEM method developed does not require a large amount of data. FFEM method only required data to determine the profile or shape of membership function. The data can usually be obtained from the opinion of expert knowledgeable in the analysis associated with inductive reasoning or a genetic algorithm. Modeling input in the form of membership function, effectively involving the epistemic uncertainty in the analysis. Although both these illustrative example are based on fuzzy approach, but the presence of a finite element method in FFEM approach allows us to use easily analysis toward the complex structure or component. In addition, the factor that affects the developed simulation of FFEM is the number of fuzzy parameters. The more obscure parameters involved in the simulation, the most conservative result of the analysis. The important decision by using fuzzy approach is the large width of interval for membership functions will give more accurate in result. The trapezoidal and triangle membership function are considered for these two illustrative examples. The result is compare and it worth mentioning that by using fuzzy value given better result in term of interval width of the membership function and also reliability values.

\section{REFERENCES}

Akpan, U.O., Koko, T.S., Orisamolu, I.R. \& Gallant, B.K. 2001. Practical fuzzy finite element analysis of structures. Finite Elements in Analysis and Design 38(2):93-111.

Behera, D. \& Chakraverty, S. 2012. A new method for solving real and complex fuzzy system of linear equations. Computational Mathematics and Modelling (23), 507-518 
Farkas, L., Moens, D., Vandepitte, D. \& Desmet, W. 2010. Fuzzy finite element analysis based on reanalysis technique, Structural Safety, 32(6): 442-448.

He, L.P., Huang, H.Z., Du, L., Zhang, X.D. \& Miao, Q. 2007. A review of possibilistic approaches to reliability analysis and optimization in engineering design, Human Computer Interaction 4553: 1075-1084.

Ozkoka, M. \& Cebi, S. 2014. A fuzzy based assessment method for comparison of ship launching methods. Journal of Intelligent and Fuzzy Systems 26: 781-791,
Savoia, M. 2002. Structural reliability analysis through fuzzy number approach, with application to stability. Computers and Structures 80(12): 1087-1102.

Tapaswini, S. \& Chakraverty, S. 2014. Non-probabilistic uncertainty analysis of forest fire model by solving hyperbolic reaction-diffusion equation. Fire safety journal, 66: 8-14.

Zimmermann, H.J. 2010. An application-oriented view of modeling uncertainty, European journal of operational research 122(2): 190-198.

Zimmermann, H.J. 2001. Fuzzy set theory and its application, Kluwer academic publisher. 